\title{
Sinomenine inhibits the expression of PD-L1 in the peripheral blood mononuclear cells of mesangial proliferative nephritis patients
}

\author{
YUE CHENG $^{1,2}$, FURONG LI ${ }^{1}$, DAIHONG WANG ${ }^{1}$, YING ZHANG $^{1}$, FAHUAN YUAN ${ }^{1}$ and JINGBO ZHANG $^{1}$ \\ ${ }^{1}$ Department of Nephrology, Xinqiao Hospital, Third Military Medical University, PLA, Chongqing 400037; \\ ${ }^{2}$ Department of Nephrology, General Hospital in Chengdu, PLA, Chengdu, Sichuan 610083, P.R. China
}

Received November 11, 2012; Accepted January 28, 2013

DOI: $10.3892 / \mathrm{mmr} .2013 .1302$

\begin{abstract}
Sinomenine has been used to treat autoimmune diseases for centuries. However, little is known about its exact mechanisms of action. Whether sinomenine has an effect on programmed death-1 (PD-1) ligands (PD-Ls) in vivo remains unclear. The present study aimed to determine the effect of sinomenine on the expression of PD-L1 and PD-L2 in peripheral blood mononuclear cells (PBMCs). A total of 25 patients with mesangial proliferative nephritis (MsPGN) were treated with sinomenine and followed up for 3 months. The expression of PD-L1 and PD-L2 was studied by using real-time RT-PCR and flow cytometric analysis, and recorded at months 0,1 and 3 within the PBMCs. The intra-renal expression of PD-L1 and PD-L2 was studied by immunohistochemistry. The results revealed that the PBMCs from the MsPGN patients expressed high levels of PD-L1 at the mRNA and protein levels compared with the healthy donors. Immunohistochemistry revealed an increased PD-L1 expression in the renal tissues from the MsPGN patients. Sinomenine was observed to have a significant effect in decreasing the PD-L1 expression in the PBMCs. The present study therefore suggests a novel mechanism for the therapeutic effects of sinomenine on MsPGN in vivo.
\end{abstract}

\section{Introduction}

Sinomenine (7,8-didehydro-4-hydroxy-3,7-dimethoxy-17-methylmorphinan-6-one) is a pure alkaloid extracted from the Chinese medicinal plant Sinomenium acutum. Previous studies demonstrated that the pharmacological properties of sinomenine included immunosuppression, anti-inflammation and arthritis amelioration $(1,2)$. Due to these beneficial effects

Correspondence to: Dr Jingbo Zhang, Department of Nephrology, Xinqiao Hospital, Third Military Medical University, PLA, 1\# Xinqiao Street, Shapingba District, Chongqing 400037, P.R. China E-mail: jingbozhang2002@yahoo.com.cn

Key words: mesangial proliferative nephritis, programmed death ligand-1, programmed death ligand-2, sinomenine and the low incidence of side-effects, sinomenine has been used for the treatment of various diseases, particularly rheumatoid arthritis (RA), systemic lupus erythematosus (SLE), renal allograft rejection and chronic glomerulonephritis (GN) (3-6). Although sinomenine is effectively used in the clinic and is gaining international popularity, its exact mechanism of action is not completely understood.

Observations from inflammatory renal diseases, including the occurrence of allograft rejection, tubulointerstitial nephritis and GN, have emphasized the key role of $\mathrm{T}$ cells in the injurious renal immune response $(7,8)$. It is accepted that signals provided by the T-cell receptor (TCR)-peptide-major histocompatibility complex (MHC) and co-stimulatory molecules are required for the optimal activation of $\mathrm{T}$ cells $(9,10)$. Selective manipulation of co-stimulatory molecules has been demonstrated to be beneficial in the treatment of autoimmune diseases, tumor and transplantation rejection $(11,12)$. The programmed death-1 (PD-1)/PD-ligand (PD-L) pathway has been extensively characterized. Based on results from PD-1 ${ }^{-/}$mice, $\mathrm{PD}-1$ may be crucial in maintaining peripheral tolerance (13). PD-L1 and PD-L2 are ligands for PD-1. The role of PD-L1 in regulating T-cell responses is controversial. However, overwhelming evidence supports the theory that PD-L1 is a negative regulator of T-cell response through either engagement with PD-1 or an unidentified receptor $(14,15)$. Nevertheless, previous studies indicated that PD-L1 was able to stimulate $\mathrm{T}$ cell activation $(16,17)$. Whether sinomenine has an immunoregulatory effect on PD-L1 and PD-L2 has, however, not been investigated.

In the present study, patients with mesangial proliferative nephritis (MsPGN), one of the most common pathological types of chronic GN, were examined. To elucidate the potential immunoregulatory properties of sinomenine, the patients were treated and then examined to determine the effects of sinomenine on the expression of PD-L1 and PD-L2 by examining peripheral blood mononuclear cells (PBMCs).

\section{Subjects and methods}

Antibodies and reagents. The mouse anti-human APC-PD-L1 (clone MIH1), PE-PD-L2 (clone MIH1), PerCP/Cy5.5-CD8a (clone RPA-T8), FITC-CD4 (clone RPA-T4) monoclonal 
Table I. Primer sequence used for real-time RT-PCR.

\begin{tabular}{lllc}
\hline Accession code & \multicolumn{1}{c}{ Name } & \multicolumn{1}{c}{ Sequence (5'-3') } & Expected length (bp) \\
\hline NM_014143 & Human PD-L1 & $\begin{array}{l}\text { Forward, TTTCAATGTGACCAGCAC } \\
\text { Reverse, GGCATAATAAGATGGCTC }\end{array}$ & 182 \\
NM_025239 & Human PD-L2 & $\begin{array}{l}\text { Forward, ATCCAACTTGGCTGCTTC } \\
\text { Reverse, CACTGTTCACTTCCTCT }\end{array}$ & 162 \\
NM_002046 & Human GAPDH & Forward, GCACCGTCAAGGCTGAGAAC & 142 \\
& & Reverse, ATGGTGGTGAAGACGCCAGT & \\
\hline
\end{tabular}

PD-L, programmed death ligand; RT-PCR, real-time polymerase chain reaction; GAPDH, glyceraldehyde 3-phosphate dehydrogenase.

antibodies (mAbs) were purchased from eBioscience (San Diego, CA, USA). The mouse anti-human PD-L1 and PD-L2 mAbs were purchased from eBioscience. The Human Erythrocyte Lysing kit was purchased from R\&D Systems (Minneapolis, MN, USA) and the Real-Time RT-PCR kit was purchased from Takara Bio Inc. (Shiga, Japan).

Patients. A total of 25 patients with MsPGN that were hospitalized in the Department of Nephrology, Xinqiao Hospital (Chongqing, China) between July 2007 and August 2008, were enrolled in the present study. The average age and gender distribution of the patients was $35 \pm 3.11$ years, 10 male/15 female, and that of the healthy donors was $30.4 \pm 0.83$ years, 4 male $/ 6$ female. No patients had been treated previously with immunosuppressant or cytotoxic drugs. All the patients enrolled had been diagnosed with primary GN with no evidence of systemic disease, including lupus nephritis, rheumatoid arthritis, other autoimmune diseases, hepatitis $\mathrm{B}$ or $\mathrm{C}$ viral infection. The diagnosis of MsPGN was based on light microscopy findings of increased mesangial cells and matrix levels [using hematoxylin and eosin (HE), Periodic acid-Schiff (PAS) and Masson staining]. Healthy donors were randomly selected from doctors and nurses in the Department of Nephrology, Xinqiao Hospital (Chongqing, China). The study protocol was conducted in accordance with procedures approved by the Human Research Ethics Board of Xinqiao Hospital and following receipt of the subjects' informed written consent. Enrolled patients received treatment with sinomenine (240 mg/day, a commonly used therapeutic dose in clinics, according to pharmacological and clinical research; purity >99\%; obtained from Zhengqing Pharmaceutical Group, Hunan, China) for $\geq 3$ months unless side-effects occurred.

Blood biochemical parameters and proteinuria detection. Blood preparations and urine aliquots were collected at months 0 , 1 and 3 during the course of the treatment with sinomenine. Creatinine, aminotransferase, and albumin levels were detected by an Olympus AU270 biochemical analyzer (Olympus, Tokyo, Japan). Complement C3 was detected by immunoturbidimetry using a Beckman Array 360 analyzer (Beckman Coulter, Miami, FL, USA). Simultaneously, the level of proteinuria was evaluated with a Sysmex UF-100 automated urinalysis analyzer (Sysmex, Kobe, Japan) and was graded semi-quantitatively from 0-3 (0, <0.15 g/l; 1, 0.15-0.3 g/l; 2, 0.3-1.0 g/1; and 3, 1.0-3.0 g/l).
Isolation of human PBMCs. Peripheral whole blood (4 ml) was collected into heparinized tubes. The human PBMCs were isolated via density gradient centrifugation using a lymphocyte separating medium according to the manufacturer's instructions. The isolated PBMCs were then lysed with TRIzol reagent (Invitrogen Life Technologies, Carlsbad, CA, USA).

$R N A$ extraction and quantitative real-time $R T-P C R$. Total RNA was extracted from the lysed cells using TRIzol reagent, following the manufacturer's instructions. Real-time PCR was performed according to the Takara two-step real-time PCR protocol. Reverse transcription (RT) of the RNA was performed using PrimeScript ${ }^{\mathrm{TM}}$ RTase (Takara). The resulting cDNA was analyzed by real-time PCR with the ABI PRISM 7500 Fast Real-Time PCR System (Applied Biosystems, Foster City, CA, USA). The primer sequences and the product size of each gene are reported in Table I. Quantification of the gene expression was performed using the $2^{-\Delta \Delta \mathrm{Ct}}$ method (18). The expression of glyceraldehyde 3-phosphate dehydrogenase (GAPDH) was used as an internal control to normalize the expression of target genes across the samples.

Flow cytometry. The PD-L1 and PD-L2 expression on the $\mathrm{CD}^{+}$and $\mathrm{CD}^{+} \mathrm{T}$ cells in the PBMCs was detected by flow cytometry. A total of $100 \mu \mathrm{l}$ whole blood was stained with corresponding antibodies and $2 \mathrm{ml}$ of H-lyse buffer was added to the blood. The surface expression of the immune molecules on the PBMCs was quantified with a FACScan flow cytometer (Becton Dickinson, Heidelberg, Germany). The FACS data were analyzed by using CELLQuestk software (BD Biosciences) and numbers indicated the mean fluorescence intensity (19). Background fluorescence was measured in the cells treated with $100 \mu \mathrm{l}$ staining buffer instead of the fluorescent antibodies. Appropriate isotype-matched antibodies from the manufacturer were used as specificity controls.

Immunohistochemistry of the renal biopsy. The intra-renal levels of PD-L1 and PD-L2 were determined by performing immunohistochemistry on the $3-\mu \mathrm{m}$ paraffin-embedded tissues from the renal biopsies of the 25 patients with MsPGN. Ten specimens obtained from pre-transplant renal biopsies (collected at Xinqiao Hospital between July 2005 and August 2008) served as the controls. Sections were incubated 
with the anti-PD-L1 (1:100) and anti-PD-L2 (1:100) antibodies overnight at $4^{\circ} \mathrm{C}$. This was followed by subsequent incubation with the secondary antibody, EnVision ${ }^{\mathrm{TM}}$ (EnVision $^{\mathrm{TM}}$ system, HRP, mouse/rabbit; Dako, Denmark) for $30 \mathrm{~min}$. Negative controls were obtained by omitting the primary antibodies or using irrelevant immunoglobulins. Reactivity was detected with a DAB Elite kit (K3465; Dako). PD-L1 and PD-L2 staining was graded semiquantitatively by noting the percentage of immunoreactive tubules (grade 0 , no reactive tubules; grade $1,<20 \%$ of reactive tubules; grade 2 , between 20 and $50 \%$ of reactive tubules; and grade $3,>50 \%$ of reactive tubules). The immunohistochemical grading was evaluated by 2 independent observers with good concordance.

Statistical analysis. All data are expressed as the means \pm SEM. The independent samples t-test and $\chi^{2}$ test were used for the continuous and categorical variables, respectively, in the comparison between the healthy donors and the MsPGN patients prior to treatment. A general linear model was used to examine the repeated measurement data. $\mathrm{P}<0.05$ was considered to indicate a statistically significant difference.

\section{Results}

Patient characteristics and laboratory data. The clinical diagnosis of MsPGN was made by the histological examination of each renal biopsy. Fig. 1 shows a patient renal biopsy slide with glomerular mesangial proliferation stained with PAS. The patients enrolled in the present study were the same individuals as in our previous study (20). The average age and the gender distribution of the patients $(35 \pm 3.11$ years, 10 male/15 female) did not differ significantly from those of the healthy donors $(30.4 \pm 0.83$ years, 4 male/ 6 female). There were no differences in the serum levels of creatinine, urea nitrogen, albumin or aminotransferase between the patients and healthy donors. There were also no differences in the values of hemoglobin and white blood cells (WBCs) between the two groups. Sinomenine was observed to have no effect on these laboratory data. In total, three patients had a transient skin rash. Varying levels of proteinuria were observed in the treated patients (the level of proteinuria was graded semiquantitatively from 0-3; 3 patients were grade 3,13 patients were grade 2 and 9 patients were grade 1 prior to treatment with sinomenine). At 1 month subsequent to initiation of the treatment with sinomenine, the proteinuria was ameliorated (11 patients were grade 2, 11 patients were grade 1 and 3 patients were grade 0$)$. At 3 months, the amelioration of the proteinuria by sinomenine was more significant ( 3 patients were grade 2, 7 patients were grade 1 and 15 patients were grade 0 ).

Changes in the expression of the PD-L1 and PD-L2 mRNA in the PBMCs. Quantitative real-time RT-PCR was used to measure the expression levels of the PD-L1 and PD-L2 mRNA in the PBMCs from the 10 healthy donors and 25 patients with MsPGN, at 0,1 and 3 months subsequent to initiation of treatment with sinomenine. As shown in Fig. 2, the PBMCs from the MsPGN patients expressed high levels of the PD-L1 $(\mathrm{P}=0.037)$ mRNA compared with the controls. There were no significant differences in the expression of PD-L2 mRNA between the MsPGN patients and the controls $(\mathrm{P}=0.627)$. The

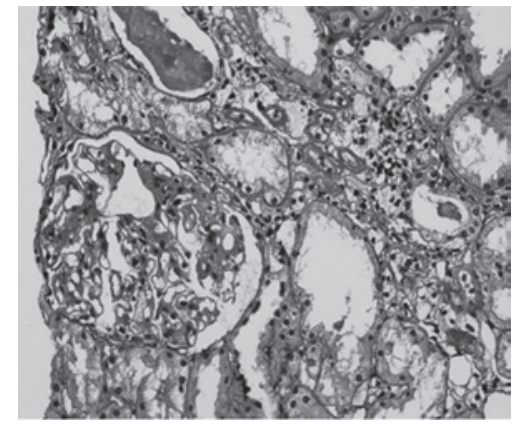

Figure 1. Light microscopy showing increased mesangial cells and matrix levels in a patient renal biopsy slide stained with PAS (magnification, x200). PAS, Periodic acid-Schiff.

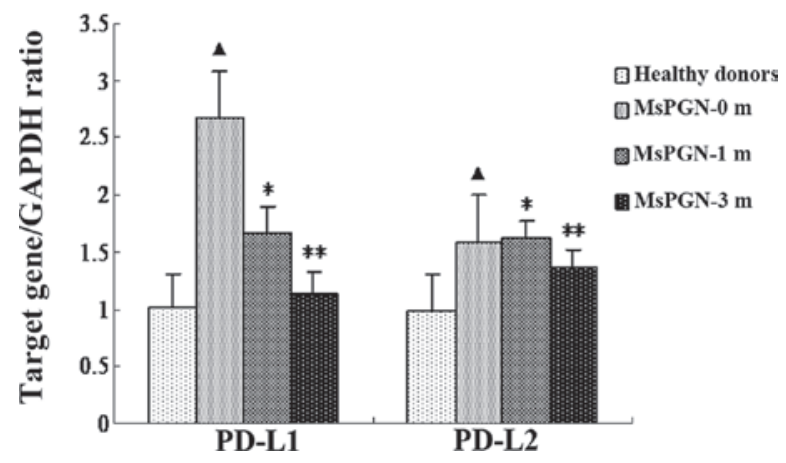

Figure 2. Quantitative analysis of the PD-L1 and PD-L2 mRNA in the peripheral blood mononuclear cells (PBMCs) from the patients with mesangial proliferative nephritis (MsPGN) and the healthy donors. The expression of PD-L1 and PD-L2 mRNA was evaluated by quantitative real-time RT-PCR as described in Materials and methods. The GAPDH housekeeping gene from the same samples was amplified as a control. The PD-L1 and PD-L2 mRNA levels from the $25 \mathrm{MsPGN}$ patients and 10 healthy donors are presented as the mean \pm SEM. MsPGN-0 m, MsPGN patients prior to treatment; MsPGN-1 m, patients 1 month subsequent to starting treatment with sinomenine; MsPGN-3 m, patients 3 months subsequent to starting treatment with sinomenine. ${ }^{\wedge} \mathrm{P}<0.05$ compared with healthy donors. ${ }^{*} \mathrm{P}<0.05$ compared with MsPGN-0 m group. ${ }^{* *} \mathrm{P}<0.01$ compared with MsPGN-0 m group. PD-L, programmed death ligand; GAPDH, glyceraldehyde 3-phosphate dehydrogenase.

expression of the PD-L1 mRNA was suppressed by sinomenine. The decrease in the PD-L1 expression was detected at $1(\mathrm{P}=0.034)$ and 3 months $(\mathrm{P}=0.002)$. However, sinomenine did not affect the expression of the PD-L2 mRNA $(\mathrm{P}=1.000)$.

Change in the expression of the PD-L1 and PD-L2 protein in the PBMCs. The expression of the PD-L1 and PD-L2 molecules on the $\mathrm{CD}^{+}$and $\mathrm{CD}^{+} \mathrm{T}$ cells in the PBMCs was investigated by using flow cytometry analysis. As shown in Fig. 3, the constitutive expression of PD-L1 was detected on the $\mathrm{CD}^{+}$and $\mathrm{CD}^{+} \mathrm{T}$ cells in the PBMCs from the MsPGN patients and the healthy donors. However, the expression of PD-L2 was hardly detected on the $\mathrm{CD}^{+}$and $\mathrm{CD}^{+} \mathrm{T}$ cells in the PBMCs from the two groups. The patients with MsPGN were observed to have an increased PD-L1 expression on the $\mathrm{CD}^{+}$and $\mathrm{CD}^{+} \mathrm{T}$ cells in the PBMCs compared with the healthy donors. Overall, treatment with sinomenine significantly inhibited the high expression of PD-L1 on the CD4 ${ }^{+}$ and $\mathrm{CD}^{+} \mathrm{T}$ cells in the PBMCs from the MsPGN patients, whereas it had no effect on the expression of PD-L2. 


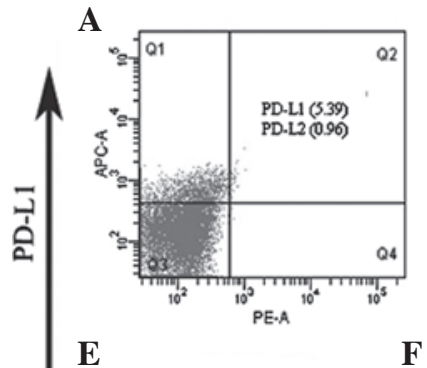

B

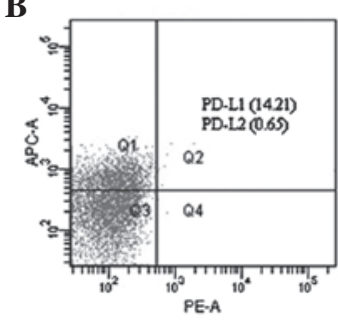

$\mathbf{E}$
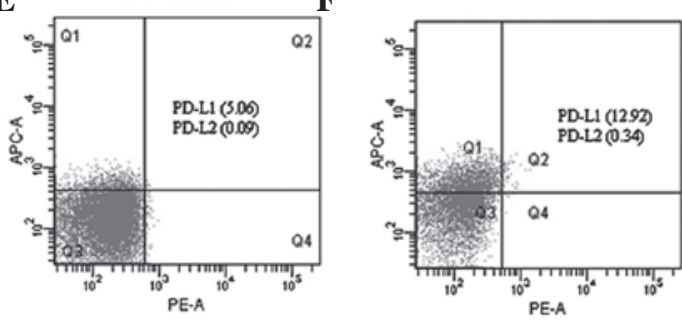

C

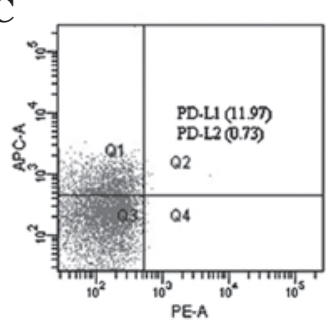

$\mathbf{G}$

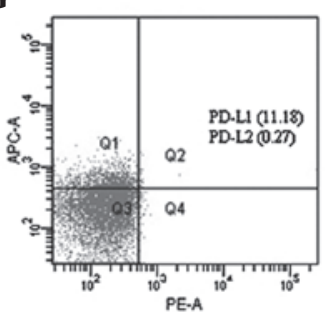

D

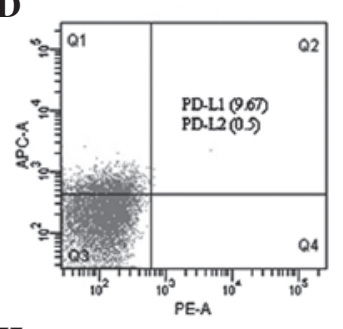

$\mathbf{H}$

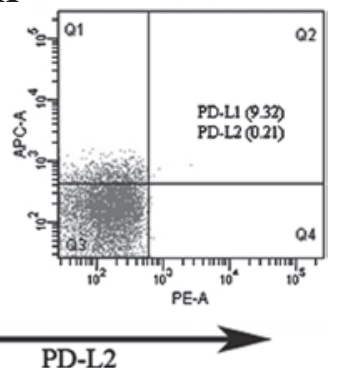

Figure 3. Expression of PD-L1 and PD-L2 on the $\mathrm{CD} 4^{+}$and $\mathrm{CD} 8^{+} \mathrm{T}$ cells in the peripheral blood mononuclear cells (PBMCs). PD-L1 and PD-L2 expression on the $\mathrm{CD}_{4}^{+}$and $\mathrm{CD} 8^{+} \mathrm{T}$ cells in the PBMCs were determined by flow cytometry using mAbs against human PD-L1 and PD-L2. Dot blots show the expression of PD-L1 and PD-L2. The percentages of the stained cells are indicated in brackets. PD-L1 and PD-L2 expression on the CD4 ${ }^{+}$T cells in the PBMCs from (A) the healthy donors, (B) the mesangial proliferative nephritis (MsPGN) patients prior to treatment, (C) the MsPGN patients at month 1 and (D) the MsPGN patients at month 3. PD-L1 and PD-L2 expression on the CD8 ${ }^{+} \mathrm{T}$ cells in the PBMCs from (E) the healthy donors, (F) the MsPGN patients prior to treatment, (G) the MsPGN patients at month 1 and $(\mathrm{H})$ the MsPGN patients at month 3. PD-L, programmed death ligand.
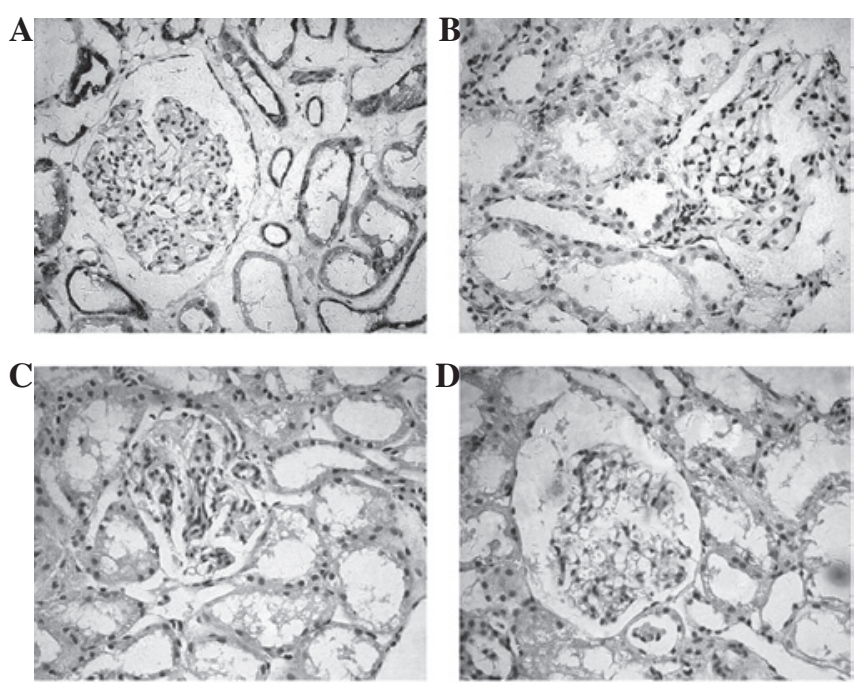

Figure 4. Detection of PD-L1 and PD-L2 expression in biopsy specimens from the normal and mesangial proliferative nephritis (MsPGN) kidneys by immunohistochemistry. (A) Biopsy from a patient with MsPGN. Significantly positive PD-L1 protein expression was present in the tubular epithelium, but absent in the glomeruli. (B) Section from a normal kidney showing no PD-L1 expression in the glomeruli and interstitium. (C) Biopsy from a patient with MsPGN. Negative staining for PD-L2 expression was detected in the glomeruli and interstitium. (D) Section from a normal kidney showing no PD-L2 expression in the glomeruli and interstitium. PD-L, programmed death ligand.

Intra-renal $P D-L 1$ and $P D-L 2$ protein expression. The expression of PD-L1 and PD-L2 in the human renal biopsy tissue samples was detected by immunohistochemistry. As shown in Fig. 4, a significant PD-L1 expression was detected in the tubular epithelium. Positive staining was detected in the cell membrane or cytoplasm or in the two together. However, the expression of PD-L1 was not observed in the glomeruli.
Table II. Intra-renal expression of PD-L1.

\begin{tabular}{lcccc}
\hline & \multicolumn{4}{c}{ No. of positive cases } \\
\cline { 2 - 5 } Group & Grade 0 & Grade 1 & Grade 2 & Grade 3 \\
\hline MsPGN $^{\text {a }}$ & 0 & 7 & 14 & 4 \\
Normal kidneys & 5 & 4 & 1 & 0 \\
\hline
\end{tabular}

PD-L1 staining was graded semi-quantitatively from 0 to 3 by the percentage of immunoreactive tubules (grade 0 , no reactive tubules; grade $1,<20 \%$ of reactive tubules; grade $2,20-50 \%$ of reactive tubules; and grade $3,>50 \%$ of reactive tubules). ${ }^{a} \mathrm{P}<0.05$ compared with normal kidneys. PD-L1, programmed death ligand-1. MsPGN, mesangial proliferative nephritis.

Patients with MsPGN were identified as having increased PD-L1 $(\mathrm{P}=0.012)$ expression in the renal biopsy tissues compared with normal individuals. The intensity levels of the PD-L1 expression in the normal or diseased renal tissues are shown in Table II. The expression of PD-L2 was not observed in the renal tissues of the MsPGN patients or the controls.

\section{Discussion}

To elucidate the potential immunoregulatory properties of sinomenine, its effect on the expression of the co-stimulatory molecules, the PD-1 ligands, by the PBMCs was investigated in the present study. The upregulation of PD-L1 was detected in the PBMCs from the MsPGN patients at the mRNA and protein levels. In contrast to PD-L1, the expression of PD-L2 in the PBMCs was hardly detected by flow cytometry in the MsPGN patients and the healthy donors, although the PD-L2 mRNA 
expression was observed. This translational suppression may be related to the different expression pattern of PD-L2, since the cell-surface expression of PD-L2 is limited to dendritic cells and macrophages, whereas PD-L1 is widely distributed in the lymphoid organs and non-lymphoid tissues $(21,22)$. We previously observed significant PD-L1 staining in the renal tubules using immunohistochemistry in diseased kidneys suffering IgA nephropathy, interstitial nephritis or lupus nephritis (19). In the present study, the high expression of PD-L1 was also detected in the tubular epithelium in the renal biopsy tissues from the MsPGN patients, suggesting that PD-L1 expression is upregulated in vivo in inflammatory kidneys. However, the expression of PD-L2 was not observed in the kidneys from the MsPGN patients. The differential expression patterns of PD-L1 and PD-L2 thus suggest different roles for these molecules in the pathogenesis of MsPGN.

While PD-L1 is associated with the negative regulation of T-cell responses via PD-1, several studies have indicated that PD-L1 was able to co-stimulate T-cell growth and cytokine production. These outcomes were produced when resting $\mathrm{T}$ cells were stimulated with suboptimal concentrations of anti-CD3 mAb, immobilized PD-L1-Ig moderately enhanced proliferation, strongly upregulated IL-10 production and modestly upregulated IFN- $\gamma$ and GM-CSF production in both human and mouse systems $(15,23)$. A noteworthy result was recorded in a study by Kanai et al (24), which observed the expression profiles of the PD-1 ligands in human inflammatory bowel disease and a murine chronic colitis model. There was a significantly increased expression of PD-L1 on the T lymphocytes, B lymphocytes, macrophage and DCs in the inflamed colons of the inflammatory bowel disease patients and the colitic mice. However, the administration of the anti-PD-L1 mAbs suppressed the wasting disease and colitis, abrogated the leukocyte infiltration and reduced the production of interferon (IFN) $-\gamma$, interleukin (IL)-2, and tumor necrosis factor (TNF)- $\alpha$ in the lamina propria of the $\mathrm{CD}^{+}$ $\mathrm{T}$ cells. These data suggested that the blockade of PD-L1 suppressed the development of chronic intestinal inflammation. In the present study, the high expression of PD-L1 in the PBMCs and renal biopsy tissues may correlate with the development of MsPGN.

Sinomenine has been widely used in China for the treatment of rheumatoid arthritis, renal allograft rejection and SLE (3-6). In the present study, the treatment with sinomenine led to significantly reduced proteinuria and elevated complement C3 levels, which demonstrated that sinomenine was able to effectively improve the clinical symptoms of the patients with MsPGN. Previous studies have demonstrated that sinomenine was able to regulate several immune functions, including i) reducing the production of TNF- $\alpha$ by activating macrophages; ii) inhibiting IL-8 and membrane (m)IL-2R and enhancing IL-6 production on the PBMCs; iii) inhibiting human $\mathrm{CD}^{+} \mathrm{T}$-cell proliferation; iv) inducing apoptosis in the synoviocytes; and v) inhibiting maturation of the monocyte-derived dendritic cells (25-30).

In conclusion, sinomenine was an effective strategy for treating MsPGN through the downregulation of PD-L1 expression. These results suggest that sinomenine may be able to regulate the $\mathrm{T}$-cell response by the inhibition of T-cell-associated PD-L1 expression.

\section{Acknowledgements}

This study is supported by the National Science Foundation of China (30570870). The authors would like to thank Senior Technician X.L. Fu from the Institute of Immunology of the PLA for her assistance in the flow cytometric analysis.

\section{References}

1. Yamasaki H: Pharmacology of sinomenine, an anti-rheumatic alkaloid from Sinomenium acutum. Acta Med Okayama 30: $1-20,1976$.

2. Deng ZS, Zhao Y, He CC, Jin J, He YM and Li JX: pH-dependent, stereoselective dimerization of sinomenine. Org Lett 10: 3879-3882, 2008

3. Zhou H, Wong YF, Wang J, Cai X and Liu L: Sinomenine ameliorates arthritis via MMPs, TIMPs, and cytokines in rats. Biochem Biophys Res Commun 376: 352-357, 2008.

4. Dai YB, Huang X, Luo ZG, Li JF, Ou YG, Yang P and Qin JP: Immunosuppressive effect of Sinomenine on ICAM-1 expression in rat renal allograft rejection. Mod J Integr Tradit Chin West Med 12: 1358-1363, 2003.

5. Yang P, Yang L and Luo Z: Effects of sinomenine on T lymphocyte subsets in rat renal allograft transplantation model. J Clin Urol 18: 620-622, 2003.

6. Feng H, Yamaki K, Takano H, Inoue K, Yanagisawa R and Yoshino S: Effect of sinomenine on collagen-induced arthritis in mice. Autoimmunity 40: 532-539, 2007.

7. Cohen RA, Bayliss G, Crispin JC, Kane-Wanger GF, Van Beek CA, Kyttaris VC, Avalos I, Yu CY, Tsokos GC and Stillman IE: T cells and in situ cryoglobulin deposition in the pathogenesis of lupus nephritis. Clin Immunol 128: 1-7, 2008.

8. Kurts C, Heymann F, Lukacs-Kornek V, Boor P and Floege J: Role of T cells and dendritic cells in glomerular immunopathology. Semin Immunopathol 29: 317-335, 2007.

9. Baxter AG and Hodgkin PD: Activation rules: the two-signal theories of immune activation. Nat Rev Immunol 2: 439-446, 2002.

10. Goronzy JJ and Weyand CM: T-cell co-stimulatory pathways in autoimmunity. Arthritis Res Ther 10 (Suppl 1): S3, 2008.

11. Loisel-Meyer S, Foley R and Medin JA: Immuno-gene therapy approaches for cancer: from in vitro studies to clinical trials. Front Biosci 13: 3202-3214, 2008.

12. Korman A, Yellin M and Keler T: Tumor immunotherapy: preclinical and clinical activity of anti-CTLA4 antibodies. Curr Opin Investig Drugs 6: 582-591, 2005.

13. Nishimura H, Okazaki T, Tanaka Y, Nakatani K, Hara M, Matsumori A, Sasayama S, Mizoguchi A, Hiai H, Minato N and Honjo T: Autoimmune dilated cardiomyopathy in PD-1 receptor-deficient mice. Science 291: 319-322, 2001.

14. Fife BT, Pauken KE, Eagar TN, Obu T, Wu J, Tang Q, Azuma M, Krummel MF and Bluestone JA: Interactions between PD-1 and PD-L1 promote tolerance by blocking the TCR-induced stop signal. Nat Immunol 10: 1185-1192, 2009.

15. Latchman YE, Liang SC, Wu Y,Chernova T, Sobel RA, Klemm M, Kuchroo VK, Freeman GJ and Sharpe AH: PD-L1-deficient mice show that PD-L1 on T cells, antigen-presenting cells, and host tissues negatively regulates T cells. Proc Natl Acad Sci USA 101: 10691-10696, 2004.

16. Dong H, Zhu G, Tamada K and Chen L: B7-H1, a third member of the B7 family, co-stimulates T-cell proliferation and interleukin-10 secretion. Nat Med 5: 1365-1369, 1999.

17. Subudhi SK, Zhou P, Yerian LM, Chin RK, Lo JC, Anders RA, Sun Y, Chen L, Wang Y, Alegre ML and Fu YX: Local expression of B7-H1 promotes organ-specific autoimmunity and transplant rejection. J Clin Invest 113: 694-700, 2004.

18. Livak KJ and Schmittgen TD: Analysis of relative gene expression data using real-time quantitative PCR and the 2[-Delta Delta $\mathrm{C}(\mathrm{T})]$ method. Methods 25: 402-408, 2001.

19. Chen Y, Zhang J, Li J, Zou L, Zhao T, Tang Y and Wu Y: Expression of B7-H1 in inflammatory renal tubular epithelial cells. Nephron Exp Nephrol 102: 81-92, 2006.

20. Cheng Y, Zhang J, Hou W, Wang D, Li F, Zhang Y and Yuan F: Immunoregulatory effects of sinomenine on the T-bet/ GATA-3 ratio and Th1/Th2 cytokine balance in the treatment of mesangial proliferative nephritis. Int Immunopharmacol 9: 894-899, 2009. 
21. delRio ML,BuhlerL, Gibbons C,Tian JandRodriguez-BarbosaJI: PD-1/PD-L1, PD-1/PD-L2, and other co-inhibitory signaling pathways in transplantation. Transpl Int 21: 1015-1028, 2008

22. Menke J, Lucas JA, Zeller GC, Keir ME, Huang XR, Tsuboi N Mayadas TN, Lan HY, Sharpe AH and Kelley VR: Programmed death 1 ligand (PD-L) 1 and PD-L2 limit autoimmune kidney disease: distinct roles. J Immunol 179: 7466-7477, 2007.

23. Tamura H, Dong H, Zhu G, Sica GL, Flies DB, Tamada K and Chen L: B7-H1 costimulation preferentially enhances CD28-independent T-helper cell function. Blood 97: 1809-1816, 2001.

24. Kanai T, Totsuka T, Uraushihara K, et al: Blockade of B7-H1 suppresses the development of chronic intestinal inflammation. J Immunol 171: 4156-4163, 2003.

25. Shu L, Yin W, Zhang J, Tang B, Kang YX, Ding F and Hua ZC: Sinomenine inhibits primary $\mathrm{CD}^{+}{ }^{+} \mathrm{T}$-cell proliferation via apoptosis. Cell Biol Int 31: 784-789, 2007.
26. He X, Wang J, Guo Z, Liu Q, Chen T, Wang X and Cao X: Requirement for ERK activation in sinomenine-induced apoptosis of macrophages. Immunol Lett 98: 91-96, 2005.

27. Wang Y, Fang Y, Huang W, Zhou X, Wang M, Zhong B and Peng D: Effect of sinomenine on cytokine expression of macrophages and synoviocytes in adjuvant arthritis rats. J Ethnopharmacol 98: 37-43, 2005.

28. Tu S, Hu Y and Lu F: Effect of Sinomenine on IL-8, IL-6, IL-2 produced by peripheral blood mononuclear cells. J Tongji Med Univ 19: 257-259, 1999.

29. Huang QC, Chen JF, Chen GX, et al: Effect of sinomenine on apoptosis of synoviocytes in CIA rats. Zhongguo Lin Chuang Kang Fu 6: 2541-2548, 2002 (In Chinese).

30. Zhao Y, Li J, Yu K, Liu Y and Chen X: Sinomenine inhibits maturation of monocyte-derived dendritic cells through blocking activation of NF-kappa B. Int Immunopharmacol 7: 637-645, 2007. 